\title{
软颗粒在液-液界面吸附及界面催化
}

杨恒权

山西大学化学化工学院, 太原 030006

\section{Soft Particles at Liquid-Liquid Interfaces for Catalysis}

\section{Hengquan Yang}

School of Chemistry and Chemical Engineering, Shanxi University, Taiyuan 030006, P. R. China. Email: hqyang@sxu.edu.cn.

Published online: April 9, 2020.
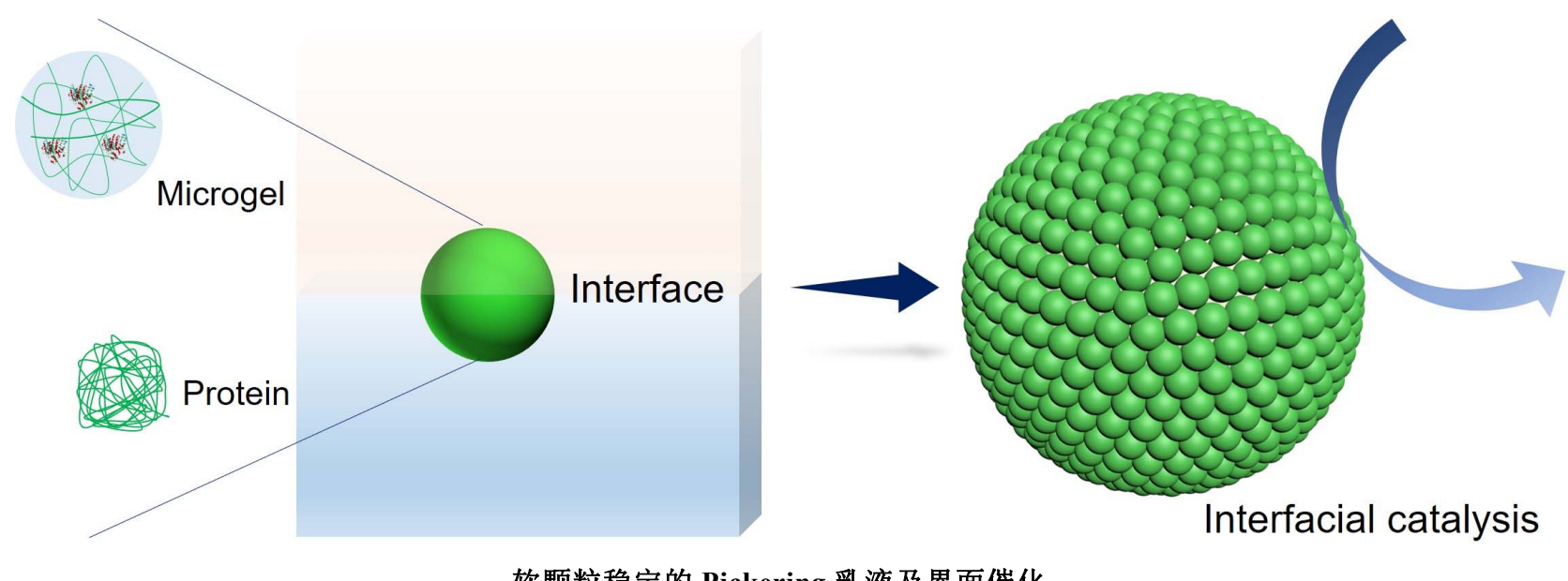

软颗粒稳定的 Pickering 乳液及界面催化。

Pickering乳液是由吸附在水油两相界面上的 颗粒稳定的乳状液, 而这些颗粒的界面脱附往往 需要很高的热力学脱附能, 使得Pickering乳液具 有良好的稳定性 ${ }^{1}$ 。相比于传统的表面活性剂稳定 的乳液, 颗粒在液液界面的存在不仅有效阻止了 乳滴间的聚结合并, 还赋予了乳液环境响应性, 如 $\mathrm{pH}$ 、温度 ${ }^{2}$ 。因此, Pickering乳液被广泛应用于医 药、催化、材料、能源、食品等领域 $3-5$ 。诸多颗粒 被证明可以作为Pickering乳液的乳化剂, 如二氧 化硅纳米球、聚苯乙烯微球、碳酸钻颗粒等。除此 以外, 软颗粒稳定的Pickering乳液越来越引起了 研究者的兴趣, 而最具代表性的便是微凝胶粒子 (microgel) ${ }^{6}$ 和蛋白质颗粒。

不同于硬颗粒, 软颗粒在乳液界面的吸附行 为更加复杂, 受到颗粒的形貌和界面形变影响。近 年来, 香港中文大学魏涛教授课题组对于微凝胶
粒子稳定的Pickering乳液和颗粒界面行为开展了 大量的研究工作 ${ }^{7}$ 。然而, 微凝胶由于其吸水溶胀 的特性, 往往只能用来制备水包油 $(\mathrm{o} / \mathrm{w})$ 型 Pickering乳液。尽管微凝胶稳定的 $\mathrm{o} / \mathrm{w}$ 型乳液在乳 液催化应用上展现了良好的应用前景, 但限制了 油溶性反应物的催化反应, 如酯化反应。近期, 香 港中文大学魏涛教授课题组联合华南理工大学和 江南大学共同研究了复合粒子协同稳定的油包水 (w/o)型Pickering乳液催化体系 ${ }^{8}$, 有效地解决了微 凝胶粒子难以用于w/o乳液界面催化的问题。

在该方法中，他们使用了疏水性的二氧化硅 纳米颗粒和具有 $\mathrm{pH}$ 响应性的微凝胶粒子, 共同作 为乳化剂制备出了 $\mathrm{w} / \mathrm{o}$ 乳液。不仅如此, 利用微凝 胶粒子的 $\mathrm{pH}$ 响应性, 还可以将脂肪酶在温和的条 件下物理封装于微凝胶聚合物网络中。而在乳化 的过程中, 该微凝胶粒子既发挥了乳液稳定的作 
用, 又将酶 “固定” 在水油界面上, 以达到高效界 面催化的目的。此类运用复合粒子协同稳定乳液 的方法有效地避免了复杂的颗粒表面改性及酶在 封装过程中失活的问题, 为微凝胶在界面催化领 域的应用提供了新的研究思路。

除了微凝胶粒子，近年来基于蛋白质的软颗 粒制备的Pickering乳液也被大量研究, 尤其在食 品领域9。然而对于这类食品级软颗粒的界面催化 研究却鲜有报道。华南理工大学尹寿伟教授和香 港中文大学魏涛教授课题组合作报道了纯酪蛋白 酸钠( $\mathrm{NaCas})$ 作为唯一乳化剂制备性能优异的 $\mathrm{pH}$ 响应 Pickering乳液体系 ${ }^{10}$, 并证明通过简单地改 变 $\mathrm{pH}$ 值便可触发 100 次循环, 这在任何蛋白质稳定 的乳液系统中从未观察到。更重要的是, 即使在敏 感和复杂的环境中, 例如饱和盐溶液 $(\mathrm{NaCl} 6.1$ $\left.\mathrm{mol} \cdot \mathrm{L}^{-1}\right)$ 或海水中, $\mathrm{NaCas}$ 稳定的Pickering 乳液也 能保持其 $\mathrm{pH}$ 响应特性。

此外, 在玉米醇溶蛋白(zein)或 $\mathrm{SiO}_{2}$ 等常规纳 米颗粒上修饰一层 $\mathrm{NaCa}$ 时, 所制备的Pickering乳 液可以稳定循环 10 次以上。 $\mathrm{NaCas}$ 的这种独特性质 可以使传统的 Pickering 乳液转变为 $\mathrm{pH}$ 响应的 Pickering乳液。基于此, 他们将具有催化活性的金 纳米团簇 $(\mathrm{Au} \mathrm{NCs})$ 负载到 $\mathrm{NaCas}$ 蛋白中, 成功实现 了蛋白质软颗粒稳定的乳液及界面催化, 为绿色 高效及可持续催化提供了新方向。

上述研究工作近期分别在 ACS Applied Materials \& Interfaces 和Chemical Science 上在线
发表 8,10 。软颗粒在Pickering乳液的实际应用方面, 尤其是界面催化领域具有极大的应用前景。

\section{References}

(1) Binks, B. P. Curr. Opin. Colloid Interface Sci. 2002, 7, 21. doi: 10.1016/S1359-0294(02)00008-0

(2) Tang, J.; Quinlan, P. J.; Tam, K. C. Soft Matter 2015, 11, 3512. doi: $10.1039 / \mathrm{C} 5 \mathrm{SM} 00247 \mathrm{H}$

(3) Wu, J.; Ma, G. -H. Small 2016, 12, 4633. doi: $10.1002 /$ smll.201600877

(4) Xiao, J.; Li, Y.; Huang, Q. Trends Food Sci. Technol. 2016, 55, 48. doi: 10.1016/j.tifs.2016.05.010

(5) Zou, H.; Ettelaie, R.; Yan, S.; Xue, N.; Yang, H. Acta Phys. -Chim. Sin. 2020, 36, 1910006. [邹后兵, Rammile Ettelaie, 间帅, 薛楠, 杨恒权. 物理化学学报, 2020, 36, 1910006.] doi: 10.3866/PKU.WHXB201910006

(6) Plamper, F. A.; Richtering, W. Acc. Chem. Res. 2017, 50, 131. doi: 10.1021/acs.accounts.6b00544

(7) Kwok, M. -H.; Sun, G.; Ngai, T. Langmuir 2019, 35, 4205. doi: 10.1021/acs.langmuir.8b04009

(8) Jiang, H.; Liu, L.; Li, Y.; Yin, S.; Ngai, T. ACS Appl. Mater. Interfaces 2020, 12, 4989. doi: 10.1021/acsami.9b16117

(9) Huang, X. -N.; Zhu, J. -J.; Xi, Y. -K.; Yin, S. -W.; Ngai, T.; Yang, X. -Q. J. Agric. Food Chem. 2019, 67, 9719. doi: $10.1021 /$ acs.jafc.9b03356

(10) Xi, Y.; Liu, B.; Jiang, H.; Yin, S.; Ngai, T.; Yang, X. Chem. Sci. 2020, 11, 3797. doi: 10.1039/C9SC05050G 